\title{
GPR resolution in Cultural Heritage applications
}

\author{
V. Pérez-Gracia, D. Di Capua, R. González-Drigo \\ Dpt. Resistencia de Materiales y Estructuras en la Ingeniería \\ EUETIB. Universidad Politécnica de Cataluña \\ Barcelona, Spain \\ vega.perez@upc.edu,daniel.di.capua@upc.edu
}

\author{
O. Caselles, L.G. Pujades, V. Salinas \\ Dpt. Ingeniería del Terreno, Cartográfica y Geofísica \\ Universidad Politécnica de Cataluña \\ Barcelona, Spain \\ oriol.caselles@upc.edu, lluis.pujades@upc.edu
}

\begin{abstract}
The non-destructive study of historical buildings, archaeological sites and other Cultural Heritage structures requires high resolution methodologies and a good knowledge of the potential of the different methods. Laboratory measurements provide valuable information about the ability to detect different targets and to determine structural problems, but these data must be compared to the results obtained in real and complex structures. In this work, we present experimental GPR measurements made in order to determine the spatial resolution under laboratory conditions. These results were compared to the data obtained in different GPR surveys applied to Cultural Heritage. The information obtained in drillings, in visual inspections and in old documentation about the historical buildings and archaeological sites is used to determine the resolution in each case.
\end{abstract}

Keywords:ground-penetrating radar, historical heritage, case studied, geophysical prospection

\section{INTRODUCTION}

Ground-penetrating radar (GPR) data obtained in the study of historical buildings is often difficult to interpret. The evaluation of historical structures and cultural heritage by means of GPR usually yields to complex radar imaging due to complexity inherent to these historical constructions. The recorded anomalies could be caused by many numbers of different elements and constructive parts. Also, the unknown restorations and changes produced during the life of the structure, introduce an important uncertainty in the data interpretation. In order to reduce the uncertainty in the GPR evaluations of the historical structures and buildings, experimental measurements under controlled lab conditions are carried out. Experimental data provide valuable information about the behavior of the GPR antennas. The results are compared to the data obtained in different case studies to determine the real possibilities in field data acquisition.

Historical structures suffer important changes during their life. Many of these changes are structural modifications usually non-documented of poorly documented. Despite of this difficulty, in this kind of studies it is necessary to obtain an imaging of the subsurface with adequate resolution. Accurate interpretation of radar data and the correct analysis of the information require a wide knowledge of the radar behavior. In this work we present several laboratory data acquisition experiments in order to determine the resolution under controlled conditions and in well-defined media. We also present several case studies. The radar images are compared to the lab data in order to determine the exact possibilities of this methodology in the study of historical structures, with a 1.6 $\mathrm{GHz}$ nominal center frequency antenna.

\section{LABORATORY DATA}

Spatial resolution depends on the characteristics of the radar signal and of the survey, the EM properties of the studied medium, and the distance from the antenna to the target. The frequencies, the number of traces or scans over the target, and the spatial antenna beam pattern are the features defining the radar signal and the survey. Experimental measures, numerical simulations and data processing indicate that, in order to detect a target, a minimum of three scans must hit on the anomalous body [1]. Resolution is the highest when the antenna is placed over the surface of the medium, and it decreases with the increase of the distance from the antenna to the surface [2]. It is also known that spatial resolution improves as attenuation increases [3] and that attenuation depends mainly on the frequencies and on the EM properties of the medium. In spite of these dependencies, horizontal resolution is often related to the footprint of the antenna (the area illuminated by the antenna), which is usually estimated as the first Fresnel zone. Different equations are utilized to approximate this zone [4]. Vertical resolution is often considered as $1 / 4$ of the wavelength [5][6], but in field measurements it is sometimes considered as $1 / 2$ of the wavelength [7][8]. Spatial resolution is largely dependent on the beam of the antenna because the footprint at different depths depends on the energy cone transmitted. The narrower the beam width, the higher the directivity of the antenna and the greater its ability to resolve closely spaced targets in the plane perpendicular to the radiation. The radiation pattern could be considered the directional radiation properties of an antenna in $3 \mathrm{D}$, with the beam being the main lobe of this radiation pattern.

\section{A. Horizontal resolution}

The well-defined media used in the laboratory measurements were water, air and sand. In these different media, two close metal bars were embedded at different depths and detected using GPR. Different bars configurations were used. Figure 1 shows an example of the radar data obtained in water with $10 \mathrm{~mm}$ diameter bars placed at a depth of $17 \mathrm{~cm}$. The two bars are detected as separated anomalies at a minimum distance between centers of $3 \mathrm{~cm}$. Reflection on the bars when distances are lower than $3 \mathrm{~cm}$ provide anomalies similar to those caused by the reflection on a single bar. This first 
evaluation allows to conclude that the distance between bars to detect them as separated anomalies was close to $0.2 \lambda$ when the depth to the anomalies was also $0.2 \lambda[4], \lambda$ being the wavelength.

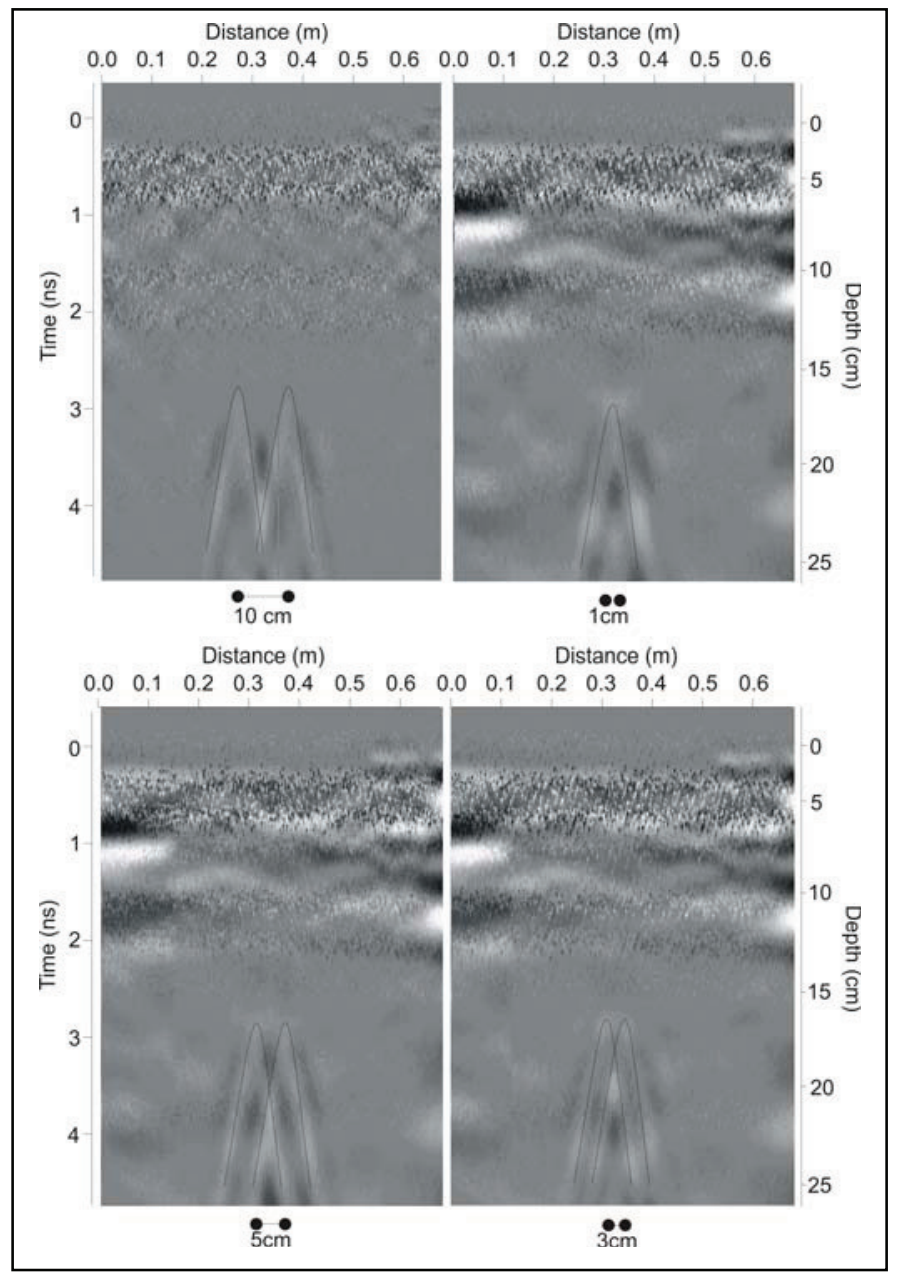

Figure 1. Radar data obtained from the reflection of the signal on two bars submerged in water separated $10,1,5$ and $3 \mathrm{~cm}$.

\section{B. Vertical resolution}

Vertical resolution was also experimentally evaluated for the $1.6 \mathrm{GHz}$ antenna in sand, water and air. A metallic surface was embedded in the different media. The depth to the metallic surface varies depending on its slope. Figure 2 shows the scheme of the experimental device in sand. Different sheet slopes were used, and the average velocity of the wave in the media was previously determined. An example of the radar data is also shown in Figure 2 in the case of a minimum depth of $2 \mathrm{~cm}$ and a slope of $25^{\circ}$. Data was filtered in order to determine clearly the reflection on the metal sheet with subtract mean trace, a time varying gain, and a DC removal.

Two different results were considered [10]. The first one corresponds to the reflected wave and the surface-coupled wave recorded as separated arrivals. In this case, the depth to the reflective surface was about $5 \mathrm{~cm}(\lambda / 2)$. The second one corresponds to when the radar data produced an overlap between the reflected and ground-coupled waves, where the arrival of the backward wave was detected. This second result was found for the shallowest part of the metallic sheet, placed at a depth of $2 \mathrm{~cm}(\lambda / 4)$.

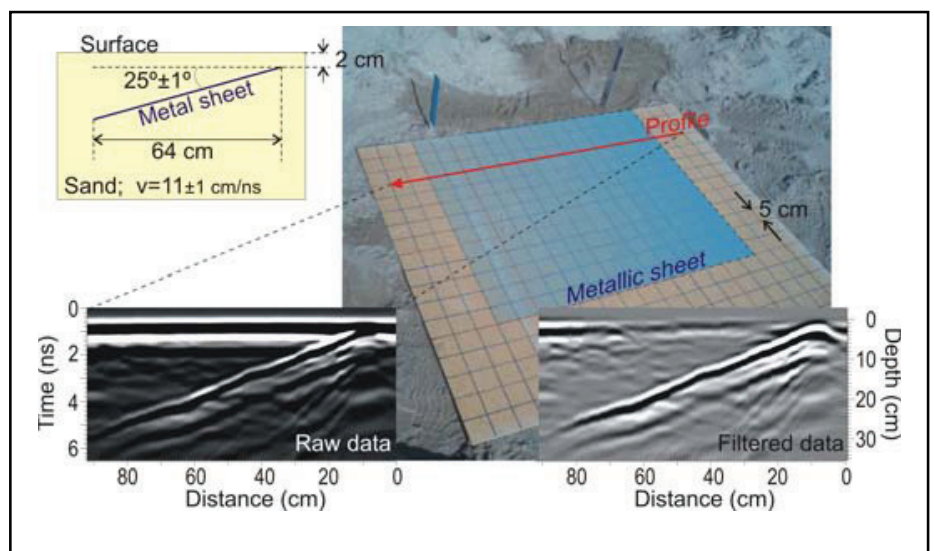

Figure 2. Radar data obtained in a metallic surface embedded in sand, presenting a slope of $25^{\circ}$, and a scheme of the experimental device.

\section{CASE STUdy 1: A MEDIAEVAl PALACE}

The building known as the Marqués de Llió palace is placed in the Montcada Street in the old district La Ribera (downtown Barcelona). This building is now composed by three different houses. The oldest house was a tower dated from the $13^{\text {th }}$ Century. This tower was expanded with a gothic gallery. Between 1538 and 1604 some remodelling was carried out, and two close houses were added to the first one. In 1705 the palace was restored and the courtyard was closed. Other non-documented changes were carried out since the building was used as school in the early XX Century.

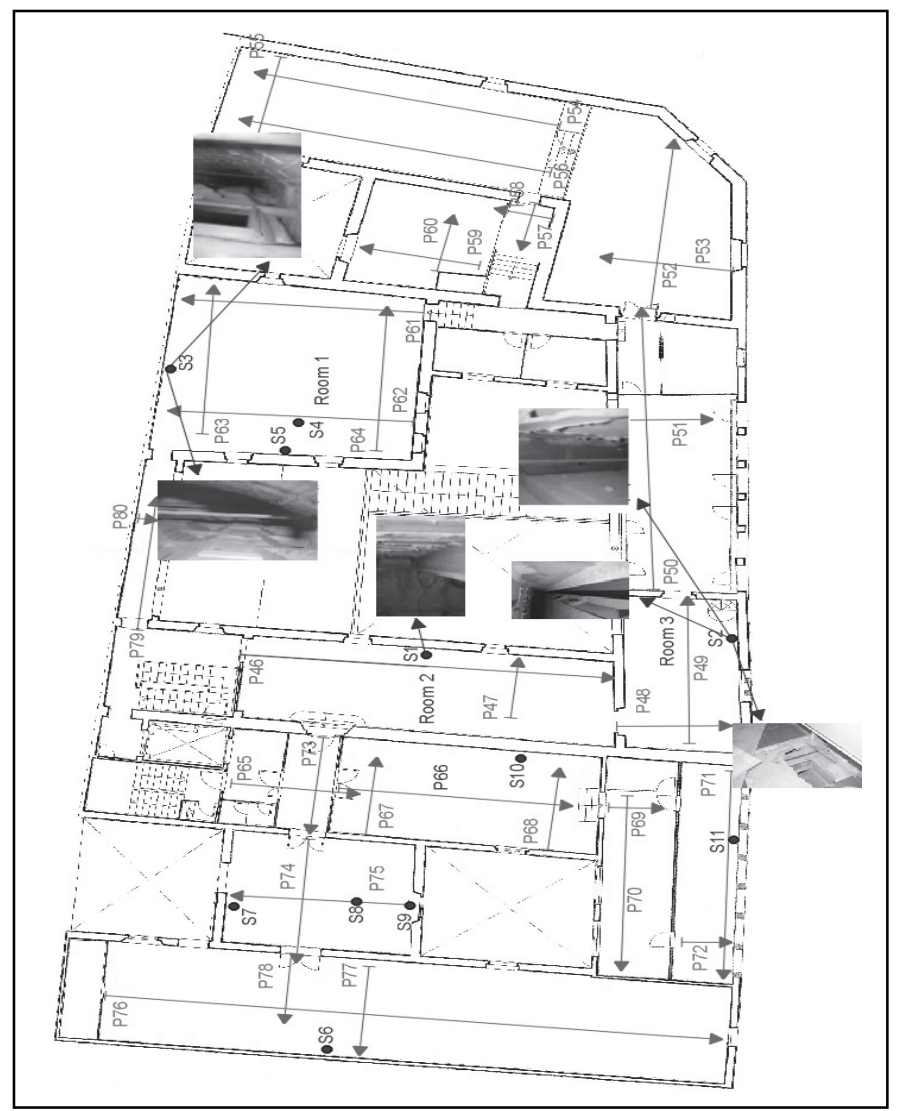

Figure 3. Second level. The rooms present diverse structures. The images correspond to the destructive tests performed after the GPR evaluation.

The last changes and restoration took place between 1965 and 1969. As a result, different constructive methods are presented in the actual rooms of the palace. The GPR survey was carried 
out in order to determine the structural solutions applied in each room [9]. The study was performed in two levels of the palace. Figure 3 shows the plant of one level and the GPR profiles.

\section{A. Beam structure}

The existence of small and double IP beams is found in room 3. Radar image shows hyperbolic anomalies with a flat and wide upper zone (see Figure 4). Two hyperbolic anomalies are also recorded later. They might be associated to the superposition effects of the reflections in the wide base of the girders that are found in the illuminated path of the antenna. The existence of two anomalies could be due to the presence of two metallic beams. The beams are represented as two single and adjacent anomalies in the migrated section.

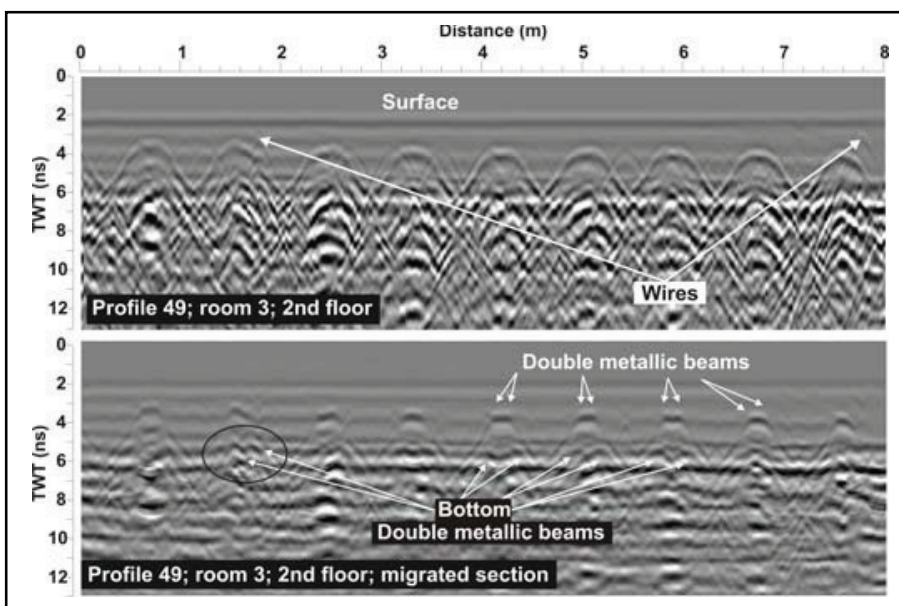

Figure 4. Radar data obtained in room 3 showing double IP beams.

The punctual drilling in this room indicates that the structure is supported by double beams, separated a free distance of two centimetres.

\section{B. Masonry arches}

Some rooms, the oldest ones, were supported by masonry arches. Later reconstructions include concrete beams to strengthen the floor. These mixed structures were detected in room 1. Figure 5 shows the radar data and the possible interpretation of the images obtained in room 1.

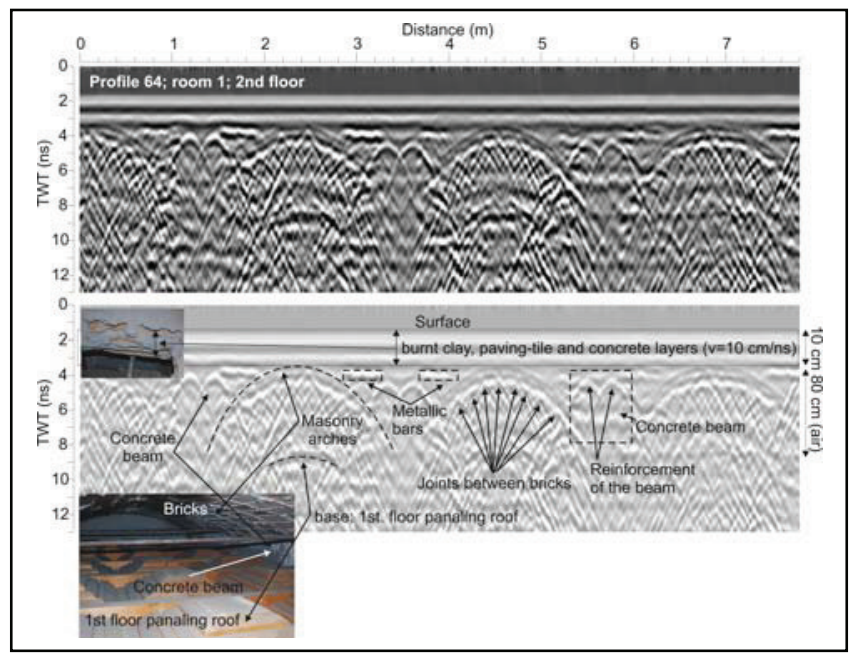

Figure 5. Radar data obtained in the room number 5 where the floor is supported by ancient masonry arches and modern concrete beams.

It is possible to observe an anomaly probably corresponding to four vaults. In this part of the image, small diffraction hyperbolas become visible most likely between each brick of the vault. These detected anomalies are not exactly true hyperbolas because the first branch is most likely produced by the sharp corner at the beginning of the second brick, and the first branch is probably caused in the sharp border at the end of the first brick.

The bricks were $15 \mathrm{~cm}$ long, as a posterior drilling indicates. The photograph in figure 5 is done in one of these punctual drillings.

\section{Stone arches}

The oldest arches build combining stones and bricks supported the central part of the palace. Similar arches are found at the entrance of the palace. Figure 6 shows the radar image obtained in this part of the building. The hyperbolas detected in the upper part are probably due to masonry boxes supporting the tiles.

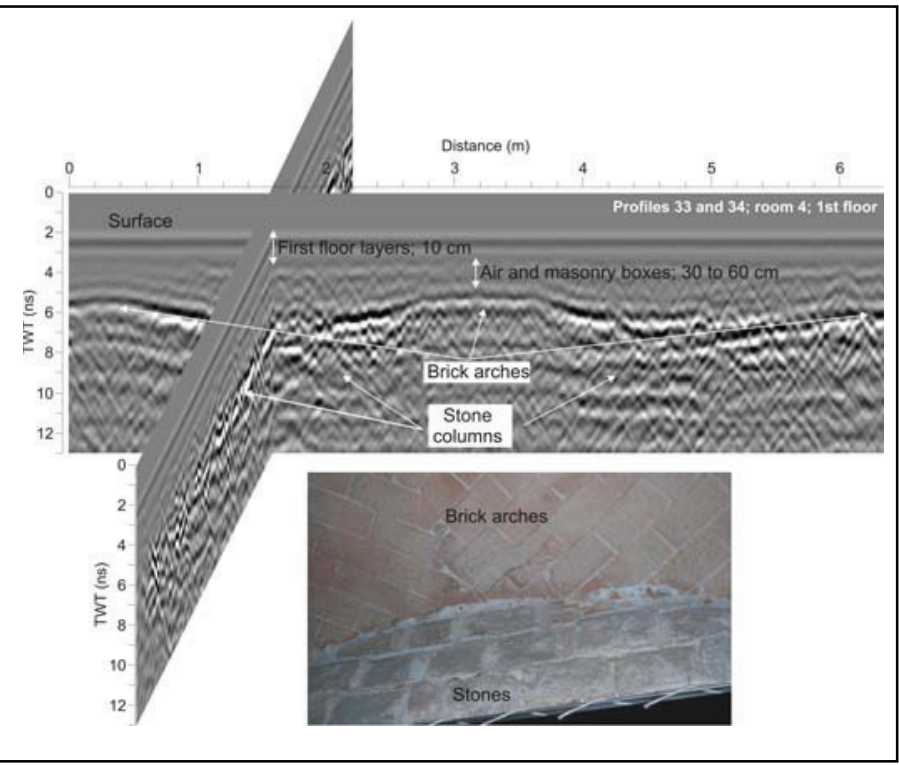

Figure 6. Radar data obtained in the first floor. The upper anomalies are produced in masonry boxes supporting the tiles. Lower anomalies are caused by the arches and columns built with stones and bricks.

\section{CASE STUDY 2. DETECTION OF ROMAN WALLS}

GPR is widely used to detect structures underneath buildings or historical sites. In these cases, it could be useful to obtain a grid of profiles, covering the whole surface. This kind of data acquisition allows the achievement of time slices and 3$\mathrm{D}$ imaging of the inner medium. Therefore, resolution is also depending on the distance between profiles, as well as on the scan per meter, the frequency, the energy distribution and other factors considered in the case of single profiles. Separation between profiles depends mainly on the size of the targets that must be detected. In this example, radar data were acquired in parallel profiles separated $50 \mathrm{~cm}$ with a $200 \mathrm{MHz}$ centre frequency ground coupled antenna. Time window was $100 \mathrm{~ns}$ and the ground was surveyed with 0.2 scans per centimeter stacking 4 adjacent traces. The wave velocity was calculated to be $8 \mathrm{~cm} / \mathrm{ns}$. Amplitude time-slice analysis was used in the areas where the grid of profiles was viable. The subsequent data is processed with GPR-Slice software [10], involved trace marker 
interpolation, time-zero shift, exponential gain correction, and band-pass filtering. No topographical corrections were needed because the site was quite leveled. The time slices were generated at 3 ns intervals using the square of the wave amplitude. They allowed easy visualization of the location, depth, size and shape of the radar anomalies corresponding to buried features. Figure 7 shows a time slice obtained between 13 and $17 \mathrm{~ns}$. This TWT corresponds to a depth near $60 \mathrm{~cm}$. In this image it is possible to observe several anomalies presenting alignments. These anomalies were caused by the reflection of the wave in the remains of roman walls and an oldest defensive wall.

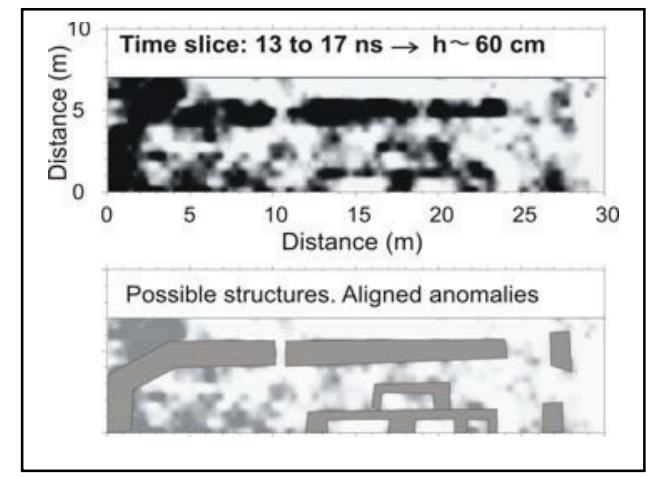

Figure 7. Amplitude distribution at a depth of about $60 \mathrm{~cm}$.

Figure 8 shows a time slice obtained at 18 to $22 \mathrm{~ns}$, and corresponding to a depth of about $80 \mathrm{~cm}$. The main anomaly observed in Figure 7 is also detected in this other time slice, but other smaller walls were also probably detected.

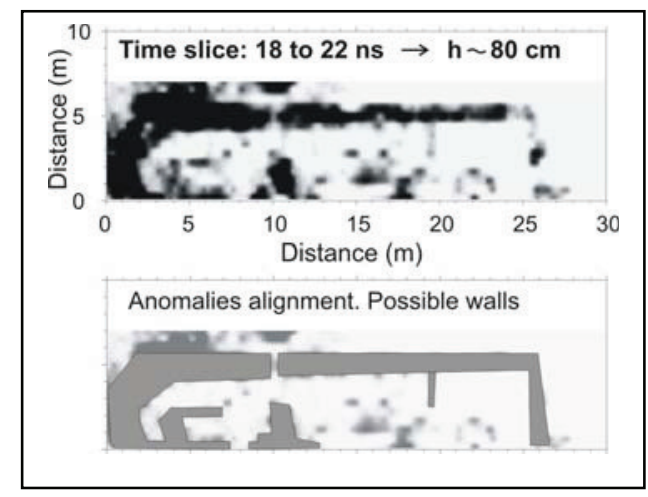

Figure 8. Amplitude distribution at a depth of about $80 \mathrm{~cm}$.

\section{CASE STUdy 3. Protomodernista Walls}

The Industrial School of Barcelona was created on March 30, 1904. In 1909 it was moved to the old textile Batlló factory of Barcelona. The Batlló textile factory's buildings were built in 1868. This factory was severely modified in order to be used as a school. The architect developed the expressive possibilities of the bricks and cut stone in the façades, vaults, windows, stairways, cornices, parapets, and other architectural elements. Another characteristic of this construction is the roof of blocked vaults, tightening and leaning on wooden girders. In some buildings, the skilled use of cast iron columns and arcades led to wide interior spaces. The whole architectural group resulted sober and balanced. Nowadays it is considered one of the most representative examples of the industrial architecture of the XIX century in Barcelona. The buildings corresponding to the early project were affected by important subsequent projects. In 1927, the remodeling of the main façade oriented toward the east was performed. As a result, three-floor buildings were added to the central area of the factory $\left(4000 \mathrm{~m}^{2}\right)$. Hinged skylights to illuminate the basements, where most of the looms were located, initially

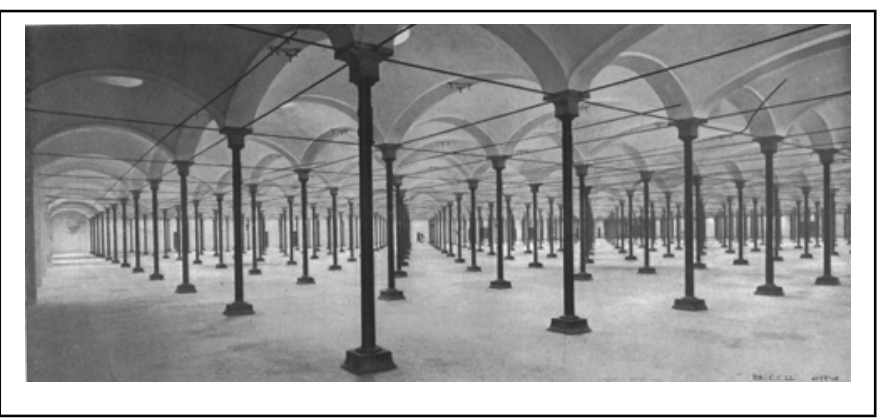

occupied this area. Long cast iron columns supported the roof of the basements (Figure 9). To support the new three-floors building, load-bearing walls built with masonry bricks substitute the old cast iron columns.

Figure 9. Old cast iron columns in the factory.

The study of the actual walls of the basement was carried out with a ground penetrating radar survey using a $900 \mathrm{MHz}$ nominal center frequency antenna [11]. The velocity in the masonry walls and columns was estimated from the GPR records, measuring the two-way travel time between anomalies that are caused by the reflection of the wave on the both sides of the walls. An average velocity of $13 \mathrm{~cm} / \mathrm{ns} \pm 1 \mathrm{~cm} / \mathrm{ns}$ was determined. The concrete walls and columns are close to $70 \mathrm{~cm}$ width or about 45 the narrower columns. Anomalies recorded in the radar data indicate the presence of the cast iron columns inside the masonry load bearing walls and columns. Figure 10 shows an example of the recorded anomalies and a photograph of the walls.

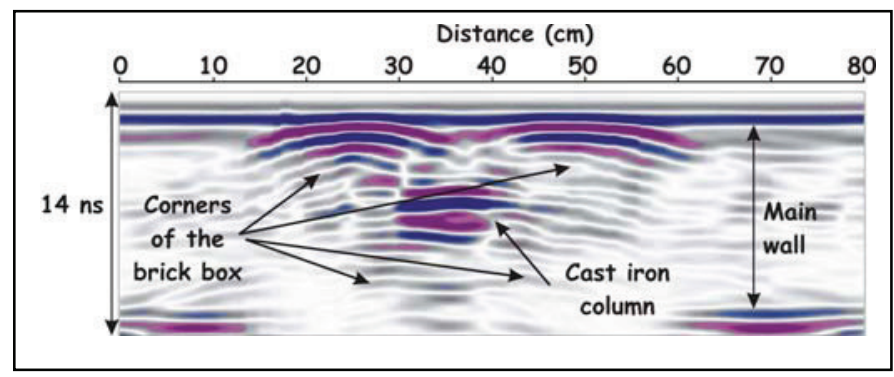

Figure 10. Example of the radar data obtained in the walls of the basement.

The diameter or the cast iron columns is about $15 \mathrm{~cm}$ and probably a masonry box is around each one of these columns. Figure 11 presents an example of the different anomalies recorded in the profiles and the possible targets existing inside the walls that most likely cause these anomalies.

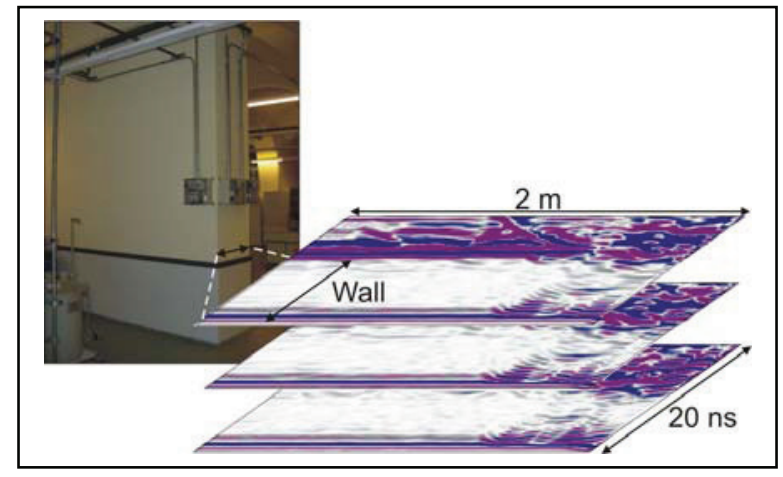


Figure 11. Radar data showing the image of the cast iron column inside the masonry load bearing wall.

\section{DISCUSSION AND CONCLUSION}

The study of cultural heritage and historical buildings by means of GPR needs a careful interpretation due to the complex radar images. This complexity is mainly due to the changes and different restorations carried out during the life of the structures. Many of these modifications are not welldocumented and the structural solutions are superimposed, and not well known. In these cases, resolution of the prospecting methods must be carefully considered. As it is shown in the different examples, a great number of anomalous targets associated to structural elements could be detected with GPR, but resolution is always lower than resolution obtained under controlled laboratory measurements. The lab measurements in well-defined media seem to indicate that horizontal resolution could be near to $0.2 \lambda$ when the targets are about $0.2 \lambda$ deep. Vertical resolution could be considered $0.25 \lambda$. Notwithstanding, resolution depends on the antenna footprint, decreasing with depth. The superimposed anomalies in the cases study make difficult the detection of small targets. In some cases it is possible to consider that radar data allows distinguishing targets separated $0.75 \lambda$ at a depth between 0.45 $\lambda$ to $0.33 \lambda$. In other cases, the elements are clearly separated in the horizontal axis when distances are higher than $\lambda$, and depths are about $0.24 \lambda$. In the historical building, separation between bricks in the vaults is also observed. Concluding, in the shallow evaluations of structures, it is possible to obtain a horizontal resolution of about $\lambda$, and a vertical resolution of about $0.5 \lambda$.

\section{ACKNOWLEDGMENT}

This study was carried out with the support of the Spanish Ministry of Science and Technology, under the project CGL2008-00869/BTE. The fifth author is supported by an FPI grant included in the research project. Authors are also grateful to the different people that help during the radar data acquisition process.

\section{REFERENCES}

[1] F.Sh.A. Ghasemi and M.S. Abrishamian, "A novel method for FDTD numerical GPR imaging of arbitrary shapes based on Fourier transform," NDT \& E Int., 40, 2007, pp. 140-146.

[2] J. van der Kruk, “'Three-dimensional GPR imaging in the horizontal wavenumber domain for different heights of source and receiver antenna," Near Surface Geoph. 2., 2004, pp. 23-29.

[3] D.J. Daniels (editor), Ground Penetrating Radar. 2nd edition, IEE Radar, Sonar and Navigation Series 15, Institution of Electrical Engineers, 2004.

[4] V. Perez-Gracia, R. Gonzalez-Drigo, D. Di Capua,“' Horizontal resolution in a non-destructive shallow gpr survey: an experimental evaluation," NDT \& E Int., 41, 2008, pp. 611-620.

[5] V. Dezelic, D.B. Apel,“ Evaluation of high frequency ground penetrating radar (GPR) in mapping strata of dolomite and limestone rocks for ripping technique," Int. J. of Surf. Mining, Reclamation and Env. 19, 2005, pp. 260-275.

[6] H. Marcak and T, Golebiowski, "Changes of GPR spectra due to the presence of hydrocarbon contamination in the ground," Acta Geoph. 56, 2008, pp. 485-504.

[7] B. Schmalz, B. Lennartz, D. Wachsmuth, "Analyses of soil water content variations and GPR attribute distributions," J. of Hydrol. 267, 2002, pp. 217-226.

[8] I.L. Al-Qadi and S. Lahouar, "Measuring layer thicknesses with GPR Theory to practice," Const. and Build. Mat. 19, 2005, pp. 763-772.

[9] V. Perez-Gracia, D. Di Capua, R. González-Drigo, L.G. Pujades, "Laboratory characterization of a gpr antenna for high-resolution testing: radiation pattern and vertical resolution," NDT \& E Int. 42, 2009, pp. 336-344.

[10] D. Goodman, "GPR-SLICE. Ground Penetrating Radar Imaging Software. User's Manual”, Geophysical Archaeometry Laboratory, California, 2004

[11] V. Perez-Gracia, O. Caselles, J. Clapes, R. Osorio, J.A. Canas, L.G. Pujades,"Radar exploration applied to historical buildings: a case study of the Marqués de Llió palace, in Barcelona (Spain),", Eng. Fail. Analysis 16, 2009, pp. 1039-1050.

[12] R. González-Drigo, V. Pérez-Gracia, D. Di Capua,’GPR survey applied to modernista buildings in Barcelona: the cultural heritage of the College of Industrial Engineering," J. Cult. Heritage 9, 2008, pp. 196-202. 